UDK: $316.7:[322: 2(4)$

Pregledni članak

Primljen 18. II. 2019.

MARTINA LuČić

Svirče, Hvar

mmlucius@gmail.com

\title{
DAS VERHÄLTNIS ZWISCHEN DER KULTURELLEN IDENTITÄT \& RELIGION DES MITTELMEERRAUMES IM REISEBERICHT REISE INS HEILIGE LAND VON ANTON PROKESCH VON OSTEN
}

\begin{abstract}
Ende März 1829 begibt sich Anton Prokesch von Osten auf eine Reise in den Orient; ins Heilige Land. Dieses Land gilt nicht nur als einer der beliebtesten Pilgerfahrtsorte des Christentums, sondern auch als Treffpunkt zwischen dem Osten und Westen im kulturellen und im religiösen Sinne.

Am Beispiel von Anton Prokesch von Ostens Reise ins Heilige Land wird in diesem Beitrag untersucht und diskutiert, was man unter den Begriffen Identität, Kultur und Religion versteht, in welchem Verhältnis die kulturelle Identität und Religion des Mittelmeerraumes stehen, bzw. ob sie sich gegenseitig beeinflussen und wenn ja, auf welche Art und Weise, welche ethnischen, kulturellen und religiösen Identitätsgruppen sich im Mittelmeerraum aus dem Reisebericht Prokeschs erkennen lassen und auf welche Art und Weise ist das Konzept des Camus' „mittelmeerischen Denkens" auf diesen Reisebericht anwendbar. Der Schwerpunkt des Beitrages liegt in der Analyse des Verhältnisses zwischen den verschiedenen kulturellen und religiösen Gemeinschaften des Mittelmeerra-
\end{abstract}


umes aus der Sicht eines österreichischen imperialen Diplomaten und Schriftstellers.

Schlüsselwörter: Europa; Mittelmeer; Orient; Identität; Religion

\section{Einleitung}

Graf Anton Prokesch von Osten (1795 - 1876) war österreichischer Diplomat, Soldat, Schriftsteller und laut Professor Dr. E. J. Görlich „der große Sohn Österreichs“. Der bedeutungsvollste Teil Prokeschs Lebens begann, als er am 19. August 1824 an Bord des österreichischen Kriegsschiffes „Il veloce“ den Hafen von Triest verließ und sich auf eine Reise in den Orient begab (cf. Prokesch von Osten, 1956, 14 - 17). In die Welt des Orients und der östlichen Mittelmeergebiete, trat Prokesch von Osten mit offenen Augen ein. Er betrachtete nicht nur das Alte, sondern auch alles Neue. Er bewunderte die Ruinen der orientalischen Städte wie z.B. Athens und Trojas und verfolgte die Politik Mehemet Alis in Ägypten, sowie die Ereignisse des griechischen Unabhängigkeitskrieges. Da sich Prokesch von Osten als fähiger Diplomat und ausgezeichneter Kenner des Orients erwiesen hat, wurde ihm das Prädikat „von Osten“ verliehen. Aus diesem Grund wurde Prokesch von Osten zum Chef des Generalstabs der österreichischen Flotte in der Levante ernannt und erhielt auch den Auftrag, die gesamte politische Korrespondenz mit Wien und mit der österreichischen Botschaft in Istanbul zu führen. Zusätzlich wurde ihm die Leitung der gesamten orientalischen Politik Österreichs im Orient anvertraut (cf. Prokesch von Osten, 1956, 19 - 21).

Die schriftstellerische Tätigkeit Prokesch-Ostens hat sehr früh begonnen und sein Talent hat sich besonders in den Schilderungen der morgenländischen Landschaft, ihrer Menschen, ihres Wesen und ihrer Zukunftsbedeutung bewiesen (cf. Prokesch von Osten, 1956, 23). Die fruchtbarste Periode seiner literarischen Tätigkeit war diejenige zwischen 1824 und 1830, als Prokesch von Osten Griechenland, die Türkei, Syrien, das Heilige Land (Palästina), Ägypten und Nubien bereiste und seine Erlebnisse in den Reiseberichten bzw. Reisetagebüchern „Erinnerungen aus Ägypten und Kleinasien“ (3 Bände; 1829 - 1831), „Das Land 
zwischen den Katarakten des Nil“ (1831), „Reise ins Heilige Land“ (1831) und „Denkwürdigkeiten und Erinnerungen aus dem Oriente" (3 Bände; 1836 - 1837) beschrieb.

Es gibt mehrere Wissenschaftler, die sich mit Leben und Werk Prokesch-Ostens beschäftigt haben, wie zum Beispiel Daniel Bertsch („Anton Prokesch von Osten (1795 - 1876). Ein Diplomat Österreichs in Athen und an der Hohen Pforte. Beiträge zur Wahrnehmung des Orients im Europa des 19. Jahrhunderts" aus 2005), Roman Míšek („Diplomat and traveller Prokesch Anton von Osten, and the Austrian attitude to the Greek uprising in the years 1826-1830", eine Dissertation aus 2008), Muhammad as-Sayyid Omar ("Anton Prokesch-Osten. Ein österreichischer Diplomat im Orient" aus 1993) und andere. Das Hauptthema von allen oben genannten Werken sind die Rolle und Bedeutung Prokesch-Ostens in den großen kulturpolitischen Ereignissen des Orients und ihre Zusammenhänge mit Europa im 19. Jarhundert.

\section{Der Orientalismus in den Habsburger Ländern}

Das Heilige Land kann man als ein perfektes Beispielland betrachten, wenn man den Verhältnis zwischen der kulturellen Identität und Religion des Mittelmeerraumes in Prokesch-Ostens Reisebericht Reise ins Heilige Land erklären möchte. Das Heilige Land gilt als Geburtsort der drei großen Weltreligionen, nämlich des Christentums, Islams und Judentums; es ist auch einer der beliebtesten Pilgerfahrtsorte des Christentums, sowie der anderen Religionen. Das Heilige Land ist also nicht nur eine geographische Bezeichnung, sondern auch ein Ort, wo die Vielfältigkeit des Orients und des Mittelmeerraumes vielleicht am meisten zum Ausdruck kommt. Bevor wir mit der Analyse Prokeschs Reiseberichts anfangen, sollten wir zuerst erklären, wie der Orient, bzw. das Osmanische Reich, dessen Teil das Heilige Land bis Ende des Ersten Weltkrieges war ${ }^{1}$, im 19. Jahrhundert von den Habsburgern angesehen wurde.

„... the region, then generally known as Palestine, remained under the sway of successive Islamic dynasties until the collapse of the Ottoman Empire at the end of World War I, ...., $<$ https://www.britannica.com/place/Israel>, (21 IV 2018). 
In den Habsburger Ländern gab es mindestens zwei Varianten des orientalistischen Diskurses. Eine Variante stellt das Bild des "fernen“ Orients (das Osmanische Reich und die Türken) dar, während die andere Variante den "heimatnahen" Orient (Bosnien und Herzegowina und die südslawischen Völker) umfasst. Die beiden Varianten wurden aus politischen Gründen von verschiedenen Protagonisten eingesetzt ${ }^{2}$ (cf. Hodkinson - Walker, 2013, 148).

In diesem Zeitraum entstand in den Habsburger Ländern auch die Dichotomie zwischen den "guten" und den "schlechten“ Orientalen. So wurden die Südslawen aus Bosnien und Herzegowina, die Christen sowie die Muslime, als "gute" Orientalen aus dem „heimatnahen“ Orient bezeichnet ${ }^{3}$ (Hodkinson/Walker, 2013: 149), während die Türken, die langjährigen Feinde der Habsburger Länder ${ }^{4}$ (Hodkinson/Walker, 2013: 150), als wenig wünschenswerte Bewohner eines „fernen“ Orients angesehen wurden (cf. Hodkinson - Walker, 2013, 149).

Da Europa in vielen Fällen als Synonym für die „Gesittung“, „Zivilisation" und die "christliche Kultur" galt und Asien, der Osten und die Türken als „kultur- und bildungsfeindliche Barbaren und Tyrannen“ bezeichnet wurden ${ }^{5}$ (cf. Hodkinson - Walker, 2013, 153), haben die

"There were at least two variants of orientalist discourse in the late Habsburg Empire. One variant represents the image of the Orient as "distant" (referring to the Ottoman Empire and the Turks), while the other conceives the Orient as "close to home" (Bosnia-Herzegovina and the Southern Slavic peoples); both variants were deployed, mainly for political reasons, by various protagonists ...."

3 „In the period of question, a dichotomy between good and bad orientals began to develop within the territories of the dual monarchy: the Southern Slavs of Bosnia and Herzegovina, Christians as well as Muslims, were perceived as the good orientals residing in the Orient "close to home", whereas the Turks living in the newly acquired provinces and beyond, also perceived as Muslims, were seen at the less desirable occupants of an ostensibly "distant“ Orient."

4 „Since the Turks had been conceived as a longstanding enemies in the Habsburg lands and beyond, this kind of demarcation provided a tool for identification and equation of new enemies inside and outside the Habsburg Empire. The Turks continued to be hated; therefore, during the last third of the nineteenth century, the image of the Turk was often used within political and religious discourses as a surrogate to represent liberals, Jews and other foes of the day."

5 „Language was used to create distance and ethno-cultural distinctions between groupings deemed "valuable" to the empire and barbarous people beyond the pale. Language proved to be a tool of cultural devaluation. In many cases, Europe was relate to "Civilisation“, 
habsburgischen "Turkophilen“ die Idee gefördert, den Orient zu „zivilisieren", indem Österreich die Rolle eines Trägers der europäischen Kultur und Sitte zugeteilt wurde. Gleichzeitig sahen sie es als ihre Herausforderung an, ,... eine Beziehung zwischen dem Osten und der abendländischen und insbesondere der deutschen Kultur im Allgemeinen herzustellen und den Handel zwischen dem Orient und Österreich-Ungarn belebend anzuregen und zu entwickeln ..." (Hodkinson/ Walker, 2013: 159-160).

\section{Kultur - Religion - Identität}

Das Verhältnis Europas gegenüber dem Orient wurde bzw. wird also oft aus der Sicht des kulturellen und religiösen Selbst und Anderen im imperialen Sinne betrachtet ${ }^{7}$. In diesem Verhältnis spielt Europa die Rolle eines Herrschers und der Orient ist nichts Anderes als seine „Erfindung" und damit sein „Untergeordnete“.

Die Wichtigkeit Prokesch-Ostens Reiseberichtes Reise ins Heilige Land liegt in der Vielfältigkeit der ethnischen, kulturellen und religiösen Identitätsgruppen, welche Prokesch von Osten als ein Europäer auf

"Gesittung", and „christliche Cultur", whereas Asia, the East, and the Turks were designated as „Barbaren“, „barbarisch“, or „Barberei“" „Tyrannen“, and „cultur- und bildungsfeindlich“ - and Islam was labeled "fanatisch“. Single protagonists on the European side are described as „edel“, „ritterlich“, „kühn“, „todesmuthig“, „umsichtig“, the other side is characterized as "roh“, „barbarisch“ and „herrschsüchtig“.

6 „Despite building bridges, the "Turkophilen“ were also clearly advancing the idea of civilizing the Orient, especially the more remote provinces of the Ottoman Empire: „Österreich's Mission als Träger europäischer Cultur und Sitte nach dem benachbarten Osten schwebt uns vor Augen." At the same time they saw it as their challenge, „die Beziehungen zwischen dem Osten und der abendländischen und besonders der deutschen Cultur im Allgemeinen zu vermitteln und dem Handel zwischen dem Oriente und Oesterreich-Ungarn insbesondere belebend anzuregen und zu entwickeln ..."

7 Wie problematisch das Verhältnis zwischen dem „christlichen“ Westen und dem „islamischen" Osten ist, diskutiert Müller-Funk in seinem Buch Die Theorien des Fremden. Laut Müller-Funk schmachtet Mohammed, der Begründer des Islams, in Dantes Divina Commedia im vorletzten Kreis der Hölle, nicht weit des letzten Kreises, in dem der Teufel selbst haust. Die muslimischen Gelehrten wie Avicenna, Avveröes und Saladin befinden sich zusammen mit den antiken Philosophen im ersten Kreis der Hölle, weil sie formal keine Christen sind. Auf diese Art und Weise wird der Islam als Konkurrent und Störfaktor angesehen (cf. Müller-Funk, 2016, 196 - 197). 
seiner Reise in das schon erwähnte Land begegnete und ihre Charakteristika in seinem Reisebericht beschrieb. Um den Zusammenhang zwischen der kulturellen Identität und Religion der Völker im Mittelmeerraum, bzw. im Heiligen Land, weiter zu diskutieren, sollten wir zuerst klären, was man unter den oben schon erwähnten Schlüsselbegriffen Kultur, Religion und Identität versteht.

Unter dem Begriff „Kultur“ versteht man die Gruppe von Institutionen, welche die Kohärenz einer Gesellschaft sicherstellt (cf. Encyclopaedia Universalis, 1989, 946). Den Begriff „Kultur“ kann man auch als integriertes Muster des menschlichen Wissens, Glaubens und Verhaltens betrachten, bestehend aus Sprache, Ideen, Glauben, Sitten, Tabus, Institutionen, Werkzeugen, Techniken, Kunstwerken, Ritualen, Zeremonien und anderen verwandten Elementen (cf. The New Encyclopaedia Britannica, vol. 3, 1997, 784). So kann man in seiner weitesten Verwendung mit dem Begriff Kultur auch all das bezeichnen, das die Menschen geschaffen haben, also alles, was nicht naturgegeben ist (cf. Brockhaus Enzyklopädie, 1990, 583).

Der zweite wichtige Begriff, das menschliche Glauben oder die Religion, kann man im allgemeinen Sinn als ein bestimmtes System oder eine Gruppe von Systemen betrachten, die Mythen, ethische und soziale Lehren, Rituale, soziale Institutionen und innere Erfahrungen und Gefühle beinhalten (cf. The New Encyclopaedia Britannica, vol. 26, 1997, 509 - 513).

Der Begriff „Identität“ [spätlat., zu lat. idem «derselbe»] lässt sich als „die völlige Übereinstimmung einer Person oder Sache mit dem was sie ist oder als was sie bezeichnet wird" definieren, aber es ist im soziologischen Sinne ein Synonym für die „kulturelle Identität" (cf. Brochkaus Enzyklopädie, 1989, 373). Die Identität einer Person ist also das Ergebnis der sozialen Vererbung, die sich aus den sozialen, religiösen und filiativen Elementen zusammensetzt (cf. Encyclopaedia Universalis, 1990, 900) und die kulturelle Identität kann man als „das im kulturhistorischem Zusammenhang erworbene (gelernte) Selbstverständnis eines Individuums, einer Gruppe oder Nation im Hinblick auf Werte, 
Fähigkeiten und Gewohnheiten ( $>$ Kultur)" definieren (cf. Brockhaus Enzyklopädie, 1990, 584).

Die Entwicklung der individuellen und gruppenspezifischen kulturellen Identität lässt sich innerhalb einer sozial (ethnische Minderheit, Klasse, Familie usw.) oder regional (deutsche, europäische usw. Kultur) definierten kulturellen Umgebung nachvollziehen. Die kulturelle Identität eines Individuums entsteht „aus dem Eingebundensein des Individuums in die kulturelle Identität eines Kollektivs und dem Bestreben nach Autonomiebewahrung der eigenen Identität". Die kulturelle Identität im traditionellen Sinne berührt auf einer positiven Identifizierung mit der Kultur einer regional (Heimat) oder klassenspezifisch (Arbeiterkultur) bestimmten Umgebung (cf. Brockhaus Enzyklopädie, 1990, 584). Im Gegensatz zu den anderen - kulturhistorischen, ethnischen, regionalen, sozialen - Aspekten wurde die Religion in dieser Definition der kulturellen Identität nicht erwähnt.

In seinem Artikel Religion in the Construction of the Cultural 'Self' and 'Other' schreibt Alan Kirp, dass die Religion im Prozess der Bildung der kulturellen Identität nicht unbedingt einbezogen werden muss. Laut Kirp gibt es heute viele neue religiösen Bewegungen, wie z.B. New Age oder verschiedene Kulte, deren Glauben keine signifikante, bzw. entweder positiv oder negativ definierende Funktion in Bezug auf die politische oder die Minderheitskultur hat. Im historischen Sinne wurden die vorindustriellen und die frühen modernen Gesellschaften jedoch durch die Religion definiert. Aus der historischen Perspektive könnte man daher schon von den spezifischen kulturellen Funktionen einer bestimmten Religion in ihrem sozialen Umfeld sprechen (cf. Kirp, 2011, 208).

Mit der Problematik der kulturellen Identität des Orients und Europas beschäftigt sich auch der englische Wissenschaftler Terry Eagleton in seinem Buch The Idea of Culture. Terry Eagleton nach besitzt der Westen keine eigene Identität, da er diese nicht benötigt, weil das Schönste daran ein Herrscher zu sein, es ist, dass sich ein Individuum 
nicht darum kümmern muss, wer er eigentlich ist. Da er ein Herrscher ist, denkt er, dass er schon weiß, wer er ist ${ }^{8}$ (cf. Eagleton, 2014, 59).

\section{Prokeschs Reise ins Heilige Land}

Prokeschs Reise ins Heilige Land begann Ende März 1829 aus Smyrna, die nämlich die Stadt İzmir in der heutigen Türkei ist ${ }^{9}$. Laut Prokesch von Osten ist das Heilige Land „... die Landstriche zwischen dem Euphrat und dem Mittelmeer, zwischen dem blühenden Syrien und dem wüsten Arabien, ..... (Prokesch von Osten, 1831, 5) Im folgenden Text wird der Zusammenhang zwischen der kulturellen Identität und Religion der Völker im Mittelmeerraum bzw. im Heiligen Land diskutiert.

Prokesch von Osten unterscheidet die Identitätsgruppen im Heiligen Land nach ihrer Religion, Herkunft, Sprache und ihrer nationalen Zugehörigkeit: die Araber, Juden, Griechen, Lateiner ${ }^{10}$, Beduinen ${ }^{11}$ usw. Laut Prokesch von Osten kommt die Bezeichnung das Heilige Land von den Juden ${ }^{12}$. Außer mit den oben schon erwähnten, begegnete Prokesch

8 „U stanovitom smislu, Zapad ne posjeduje vlastiti zaseban identitet, jer mu on i ne treba. Ljepota činjenice da se bude vladar nahodi se u tome što se pojedinac ne treba brinuti o tome tko on jest, budući da živi u opsjeni kako to već zna."

9 „Ich ging zu Ende März von Smyrna mit der k.k. Korvette Veloce unter Segel“ (Prokesch von Osten, 1831, 10).

10 Wenn er in seinem Reisebericht schreibt, dass die Besatzung des Schiffes k.k. Korvette Veloce gegen den 12. April „die Anker auf der Rhede von Kaipha“ warf (Prokesch von Osten 1831: 18), erklärt er zusätzlich, dass die verschiedenen Völker diese Stadt auch unterschiedlich nennen: „Die Araber nennen es Hipha, die Juden Kepha oder auch Hepha, die Griechen und Lateiner meist Kaipha oder Kaiphas." (Prokesch von Osten, 1831, 18). Anhand dieses Zitats könnten wir schlussfolgern, dass sich alle diese Identitätsgruppen in der Stadt Kaipha treffen oder dort leben und die schon erwähnte Stadt im Geiste der eigenen Sprache und Herkunft nennen.

${ }^{11}$ Die Beduinen im Heiligen Land sind Hirten und gehören nicht in die Klasse jener rein-arabischen Wanderhorden wie z.B. in Nubien oder in Ägypten: „Die Beduinen waren aus den Stämmen Anasi (aus der Wüste), Beni-Sohor (am Jordan), Szefech (am Thabor) und aus turkomannischen Horden. Diese Beduinen sind durchaus Hirten, und gehören nicht in die Klasse jener rein-arabischen Wanderhorden, die man in der Syrte, in Ägypten, in Nubien und im eigentlichen Arabien findet ..." (Prokesch von Osten, 1831, 137).

12 „Diese Bezeichnung kommt von den Juden, in so ferne dies Land als das vorzugsweise von dem Gotte, den sie anbeten, sich und ihnen erwählte betrachten; sie gilt den Christen, in so ferne sie Söhne des Judenthumes sind, und hauptsächlich, weil in diesem Lande, wie auf einem großen Altar, das Opfer ihres Heilandes vollbracht wurde." (Prokesch von Osten, 1831, 
von Osten im Heiligen Land auch weiteren Identitätsgruppen: die Türken, Katholiken, Griechen, Armenier und Mohammedaner ${ }^{13}$. Aus dem Reisebericht Prokesch-Ostens erfahren wir also, dass das Heilige Land damals meistens von Mitgliedern der drei abrahamitischen oder abrahamischen Religionen, also dem Judentum, Christentum und Islam, bewohnt war. Die Juden, Christen und Muslimen sind daher eng in einem kulturhistorischen und religiösen Zusammenhang verbunden. Prokesch von Osten betrachtet das Zusammenleben von den Christen, Juden, Muslimen, Arabern, Türken, Griechen und anderen Völker im Heiligen Land nicht nur aus der Sicht eines übergeordneten Abendländers.

\footnotetext{
5). Ältere Bezeichnungen für dieses Land sind auch „das Land“, „das gelobte Land“ und „das Land Israel“. Diese Bezeichnungen kann man auch in den Büchern des Alten Testaments finden, in den Büchern Samuels und der Könige. (Prokesch von Osten 1831: 6) Weitere Bezeichnungen für das Heilige Land, die, Prokesch von Osten nach, „... oft für den Begriff des gesamten Landes gebraucht, aber eigentlich nur Theilen desselben gehörig, ..." (Prokesch von Osten, 1831, 7) sind Land Kanaan, Gilead und Judäa. Unter Kanaan wurde im engeren Sinne das Land westlich dem Jordan verstanden, unter Gilead häufig das Land östlich dem Jordan und das Judäa bezeichnet eigentlich den von dem Tribus Juda bewohnten Landstrich. Prokesch von Osten betont, dass sie „Neueren“ am häufigsten die Bezeichnung Palästina verwenden und darunter oft nur das eigentliche Kanaan verstehen (Prokesch von Osten, 1831, 6 - 8). Hätten wir die Identitätsgruppen aus dem Zitat über die Einwohner der Länder Kanaan, Gilead und Judäa weiter analysiert, würden wir wahrscheinlich schlussfolgern, dass die Bezeichnung „Israeliten“ die einzige ist, die wir in einen konkreten geographischen Raum unterbringen können. Die Israeliten wären logischerweise die Einwohner von Israel. Ihrer geographischen Lage nach könnten wir auch schlussfolgern, dass diese z.B. Juden oder Christen sein könnten. Aber was würden wir von „den Moabitern, den Stämmen „Ruben, Gad und halb Manasse" und dem Tribus Juda" sagen, wenn wir z.B. keine Historiker oder Theologen, bzw. Bibelkenner sind? Aus ihren Bezeichnungen lässt sich nicht erkennen, woher genau sie kommen oder welchen Weltreligion sie angehören.

${ }_{13}$ Auf dem Weg zum Heiligen Grab reiste er mit einer Karavane, die „... aus Katholiken, Griechen, Armeniern und Mohamedanern ..." bestand (Prokesch von Osten, 1831, 39), der Ort Errit-el-Enneb, welchen er besuchte, war „... von den Arabern bewohnt, die uns [ihnen] freundlich entgegen kamen ..." (Prokesch von Osten, 1831, 41), in der Kirche zum Heiligen Grabe traf er Türken, die „Zöllner und Wächter des Tempels“ (Prokesch von Osten, 1831,52), und in Betlehem wohnten damals ,... über 1000 Katholiken, an 100 Griechen, 30 armenische und 40 türkische Familien." Das Volk in Betlehem war "gut bewaffnet" und es „trug reiche Kleider" und daher hielt es Prokesch von Osten für muselmänisches Volk, aber „... es bestand meist aus Christen, denn diese haben das Recht, Waffen zu tragen ..." (Prokesch von Osten, 1831, 113).
} 
Das Verhältnis, bzw. die Begegnung zwischen dem Osten und Westen (Europa und Orient) muss aber nicht nur durch ihre kulturellen Unterschiede bestimmt werden. Vielleicht ist das der Grund, warum Camus, so Neil Foxlee, den Orient, bzw. den Mittelmeerraum als Treffpunkt zwischen dem Osten und Westen sah (cf. Foxlee, 2010, 273). Dieses Treffen kann und soll vor allem durch gegenseitigen Respekt und Verständnis bestimmt werden. Eine solche Betrachtung des Treffens zwischen dem Osten und Westen im Orient bzw. im Mittelmeerraum ist dem Camus' Betrachtung von dieser Problematik sehr ähnlich. Dies führt uns zu einem weiteren Prinzip, welches wir in diesem Vortrag diskutieren werden - dem Prinzip des sogenannten mittelmeerischen Denkens Albert Camus'.

In seinem historisch-politisch-philosophischen Essay L'Homme revolté (dt. Der Mensch in der Revolte) aus 1951, beschäftigt sich so Camus mit den Themen des Mordes und Revoltes in Europa und in dem Mittelmeerraum aus der Sicht der historischen Kriege und kolonialistischen Bestrebungen Europas in Bezug auf den Mittelmeerraum ${ }^{14}$.

Neil Foxlee stellt dem Griechisch-mittelmeerischen oder mittelmeerischen Denken Camus' einen Korrekturgedanken entgegen ${ }^{15}$ (cf. Foxlee, 2010, 51), bzw. wurde das Griechisch-mittelmeerische Denken als die authentische Rebellion gegen die mörderischen Feldzüge des Westens, bzw. Europas bezeichnet ${ }^{16}$ (cf. Foxlee, 2010, 266). Laut Albert Camus ist das Europa „von Gespenstern und Maschinen bevölkert“ (Camus,

14 Der Mord seines Vaters, der im Ersten Weltkrieg getötet wurde, und der Algerienkrieg, der als Folge der kolonialen Ungerechtigkeit ausbrach, hatten einen großen Einfluss auf das Leben und Werk Camus'.

${ }^{15}, \ldots$ This would most famously be expressed in 'La pensée de midi' ('Noonday Thought'), the concluding part of Camus's 1951 historico-politico-philosophical essay L'Homme revolté, in which he proposed Greco-Mediterranean thought as a corrective to what he saw as the disastrous influence of German ideology, in the shape of both Marxism and Nazism."

$16 \ldots$,...This bias is equally apparent in L'Homme revolté (1951), notably in its final part, 'La pensée de midi' and the penultimate section of the same name. At the end of what he described as 'this long survey of rebellion and nihilism' (III, 313), ${ }^{11}$ Camus proposed Greco-Mediterranean measure, a philosophy of limits and 'noonday thought' as characteristic of authentic rebellion, as opposed to the murderous perversions of rebellion epitomized by German ideologies of excess (demesure) - Marxism and Nazism. ..." 
2016, 356) und ihr Geheimnis ist, dass es "das Leben nicht mehr liebt ${ }^{{ }^{17}}$ (Camus, 2016, 398).

Im kolonialen Kontext könnte, Camus nach, ein Treffen zwischen dem Osten und Westen, bzw. Europa und dem Orient niemals zu den gleichen Bedingungen stattfinden. Camus nach könnte die Begegnung der Europäer und der „Araber“ als potenziell bereichernd für die Europäer angesehen werden, da sie auf diese Art und Weise viel über die Vielfältigkeit der Mittelmeerkultur lernen könnten ${ }^{18}$ (cf. Foxlee, 2010, 273).

Diese weit fortgeschrittene Idee über die Begegnung zwischen den Europäern und den „Arabern“ hat Prokesch von Osten fast ein Jahrhundert vor Camus erkannt. Durch diese Begegnung können die Europäer viel über die Vielfältigkeit der Mittelmeerkultur lernen: ihre Geschichte, Kultur, Identität, Religion und Zusammenleben von den unterschiedlichen Völkern, die in diesem Raum leben. Als imperiale Diplomat betrachtet Prokesch von Osten den Orient nicht von dem imperialistischen Standpunkt und darin liegt die Wichtigkeit seines literarischen Nachlasses.

${ }_{17}$ Albert Camus schreibt in seinem Essay, dass die Geschichte der ersten Internationale, in der der deutsche Sozialismus gegen das freiheitliche Denken der Italiener, Spanier und Franzosen ankämpft, nämlich die Geschichte zwischen der deutschen Ideologie und dem mittelmeerischen Geist ist. Camus nach bestand der eigentliche Konflikt letztes Jahrhunderts nicht so sehr zwischen der deutschen Ideologie, der christlichen Politik und der Geschichte und der christlichen Politik, die auf eine gewisse Weise Komplicen sind, als vielmehr zwischen den deutschen Träumen und der mittelmeerischen Tradition. Das Sonnendenken, bzw. die Kultur mit dem doppeltem Gesicht, erwartet inmitten der europäischen Nacht ihre Morgendämmerungund beleuchtet schon die Wege einer echten Überlegenheit (cf. Camus 2016, $390-392)$.

$18 \ldots$ If Camus defined one aspect of the identity of the Mediterranean by opposing it to Northern Europe, he defined another aspect of the region's identity by presenting the Mediterranean - and specifically North Africa - as the meeting-point of East and West, of 'Arabs' and Europeans. In a colonial context, of course, any such meeting that took place could never be on equal terms. In theory, however, as we have seen, Camus saw the encounter with 'Arabs' as potentially enriching for Europeans, telling the audience of his lecture that the East with which they were in direct contact had much to teach them about the diversity of Mediterranean culture." 


\section{Schlussfolgerung}

Aus dem Reisebericht Reise ins Heilige Land lässt sich erkennen, dass die Prokeschs Neugierde und der Reiz eines ihm unbekannten Landes und ihrer Menschen als Zweck für seine Reise gelten, was in den Reiseschilderungen der europäischen Schriftsteller des 19. Jahrhunderts ein sehr häufiges Thema war. Die Besonderheit dieses Reiseberichtes liegt in der Tatsache, dass sein Autor ein Österreicher bzw. ein Abendländer ist, welcher einen imperialistischen Hinterland hatte, was aber für das Thema dieses Beitrages und die Entwicklung des historischen Verhältnisses zwischen dem Westen und Osten besonders interessant und wichtig ist.

Anhand der theoretischen Grundlagen und den ausgewählten $\mathrm{Zi}$ taten aus dem Reisebericht Reise ins Heilige Land kann man am Ende dieses Beitrages schlussfolgern, dass Prokesch von Osten die Identitätsgruppen im Heiligen Land nach ihrer ethnischen, geographischen, historischen, sprachlichen und religiösen Zugehörigkeit unterscheidet. Es kann sein, dass man in einem allgemeinen Sinn die kulturelle Identität ohne den Einfluss von der Religion bilden kann, aber in Bezug auf diesen Reisebericht ist das nicht der Fall. Die kulturelle Identität von den Christen, Juden und Muslimen - drei Hauptidentitätsgruppen im Heiligen Land laut dem Reisebericht Prokeschs - ist durch ihre Religion stark definiert.

Hier stellt sich die Frage, wie wird die (kulturelle) Identität Europas, bzw. des Orients bestimmt? Spielt die Religion auch in diesem Verhältnis eine Rolle und wenn ja, welche? Das Verhältnis zwischen Europa und dem Orient ist ein sehr problematisches Thema, da der Orient meistens als „islamisch" und der Okzident, also Europa oder der Westen, als "christlich“ bezeichnet wurden. Wenn Müller-Funk in seinem Buch Theorien des Fremden über diesem Verhältnis schreibt, bezieht er sich auf Edward Said und seinen Orientalismus. Laut Müller-Funk ist der Orientalismus eine westliche Diskursmacht-Formation, mit der die europäische Kultur der nachaufklärerischen Periode im Zustand war, Kulturen des Nahen Ostens soziologisch, politisch, ideologisch, 
militärisch, imaginativ und wissenschaftlich zu kontrollieren, zu leiten und zu produzieren (cf. Müller-Funk, 2016, 199). Prokesch von Osten betrachtet aber den Orient nicht im Sinne Saids, sondern versucht er die Kulturen, bzw. die Identitätsgruppen des Nahen Ostens und das Verhältnis zwischen ihnen so objektiv wie möglich zu beschreiben.

Die zwei Varianten des orientalistischen Diskurses in den Habsburger Ländern, bzw. die Dichotomie zwischen den "guten“ und den „schlechten“ Orientalen, lassen sich im Reisebericht Prokesch-Ostens nicht erkennen. Prokesch von Osten betrachtet die Identitätsgruppen im Heiligen Land nicht als die „schlechten“ Orientalen oder als die „kultur- und bildungsfeindliche Barbaren und Tyrannen" aus dem "fernen“ Orient.

In Anbetracht der gegenwärtigen Situation im Nahen Osten scheint es, dass Camus und Prokesch Recht hatten. Der ganze Raum, in welchem Mittelpunkt sich das Heilige Land befindet, wurde heute durch Kriege zerstört, wie zum Beispiel das von Prokesch von Osten erwähnte „blühende“ (Prokesch von Osten, 1831, 5) Syrien.

Wie dies Prokesch von Osten in seinem Reisebericht Reise ins Heilige Land dargestellt hat, wäre die Hauptrolle des Heiligen Landes in diesem problematischen Verhältnis, bzw. Zusammentreffen zwischen Europa und dem Orient, die Unterschiede zwischen diesen zwei Welten zu vermindern und die gemeinsamen Konflikte zu lösen, sowie zu beweisen, dass es doch möglich wäre, in einer Einheit zusammen zu leben (wie z.B. Prokesch von Osten, 1831, 18 oder Prokesch von Osten, 1831, 113).

\section{Quellen}

- Prokesch von Osten, Anton (1831) Reise ins Heilige Land. Im Jahr 1829, Carl Gerold, Wien.

- Prokesch-Osten, Anton (1956) Abendland-Morgenland. Eingeleitet und ausgewählt von E. J. GöRLICH, Stiasny Verlag, Graz - Wien. 


\section{Literaturverzeichnis}

- „Albert Camus“, Britannica, <https://www.britannica.com/biography/Albert-Camus $>$, (17 IX 2017).

- Bertsch, Daniel, „Anton Prokesch von Osten“, H-Soz-Kult, <https://www.hsozkult.de/publicationreview/id/reb-9206> (6 II 2020).

- Brockhaus Enzyklopädie in vierundzwanzig Bänden. 19., völlig neu bearbeitete Auflage. Zehnter Band. HERR - IS (1989), F. A. Brockhaus, Mannheim.

- Brockhaus Enzyklopädie in vierundzwanzig Bänden. Neunzehnte, völlig neu bearbeitete Auflage. Zwölfter Band: KIR-LAG und zweiter Nachtrag (1990), F.A. Brockhaus, Mannheim.

- Camus, Albert (2016) Der Mensch in der Revolte. Essays, 31 ed, Rowohlt Verlag GmbH, Reinbek bei Hamburg.

- Encyclopaedia Universalis. Corpus 11. Guerre et paix - Incendies (1990), Encyclopaedia Universalis, Paris.

- Encyclopaedia Universalis. Corpus 6. Climatologie - Cytologie (1989), Encyclopaedia Universalis, Paris.

- Foxlee, Neil (2010) Albert Camus's 'The New Mediterranean Culture': A Text and its Contexts (Modern French Identities), Peter Lang AG - Internationaler Verlag der Wissenschaften, 1 ed. (6 X 2010).

- Hodkinson, James - Walker, John - Mazumdar, Shaswati - Feichtinger, Johannes (2013) Deploying Orientalism in Culture and History. From Germany to Central and Eastern Europe, Camden House, Rochester, New York.

- „Israel“, Britannica, https://www.britannica.com/place/Israel (21. IV. 2018)

- Kaštelan, Jure - Bonaventura, Duda (1986) Biblija, Stvarnost, Zagreb. 
- Kirp, Alan, „Religion in the Construction of the Cultural 'Self' and 'Other', ENDC Proceedings, vol. XIV, 2011, p. 197 - 222, $<$ http://www.ksk.edu.ee/wp-content/uploads/2012/12/KVUOA_ Toimetised_14_9_alar_kilp.pdf>, (9 IX 2017).

- MíšEK, Roman, Diplomat and traveller Prokesch Anton von Osten, and the Austrian attitude to the Greek uprising in the years 1826183o, Univerziteta Karlova Prag, <https://is.cuni.cz/webapps/zzp/ detail/24477?lang=en $>$ (6 II 2020).

- Müller-Funk, Wolfgang (2016) Theorien des Fremden: Eine Einführung, UTB GmbH, Wien.

- The New Encyclopaedia Britannica (1997) vol. 3, Micropaedia.

- The New Encyclopaedia Britannica (1997) vol. 26, Micropaedia. 


\title{
THE RELATIONSHIP BETWEEN THE CULTURAL IDENTITY \& RELIGION OF THE MEDITERRANEAN IN THE TRAVELOGUE REISE INS HEILIGE LAND BY ANTON PROKESCH VON OSTEN
}

\begin{abstract}
At the end of the March 1829, Anton Prokesch von Osten went on another journey to the Orient; this time to the Holy Land. This country is not only considered as one of the most popular Christian pilgrimage sites, but also as a meeting point between the East and the West in the cultural and the religious sense.

Therefore, we will try to explain in this article what is meant by the terms identity, culture and religion, what is the relationship between the cultural identity and the religion of the Mediterranean, whether they influence each other and if so, in which way, which ethnic, cultural and religious identity groups of the Mediterranean can be identified in the Prokesch's travelogue and also in which way the concept of Camus' "noonday thought" can be applied to this travelogue. The main focus of this article is put on the analysis of the relationship between the different cultural and religious identity groups of the Mediterranean from the point of view of this Austrian diplomat and writer.
\end{abstract}

Keywords: Europe; Mediterranean Basin; Orient; identity; religion 


\title{
VEZA IZMEĐU KULTURNOGA IDENTITETA I RELIGIJE MEDITERANA U PUTOPISU PUTOVANJE U SVETU ZEMLJU ANTONA PROKESCHA VON OSTENA
}

\section{Sažetak}

\begin{abstract}
Krajem ožujka 1829. Anton Prokesch von Osten otišao je na svoje drugo putovanje na Orijent; ovaj put u Svetu Zemlju. Ova se zemlja smatra jednim od najpopularnijih kršćanskih hodočasničkih mjesta, ali i sastajalištem Istoka i Zapada u kulturnome i religioznome smislu.

Stoga ćemo u ovome radu pokušati objasniti što se podrazumijeva pod pojmovima identitet, kultura, religija, koja je veza između kulturnoga identiteta i religije Mediterana, utječu li jedno na drugo i, ako jesu, na koji način, koje se etničke, kulturne i religiozne grupe identiteta Mediterana se mogu identificirati u Prokeschevome putopisu i kako se koncept Camusove "podnevne misli“ može primijeniti na ovaj putopis. Rad je usredotočen na analizu veze između različitih kulturnih i religioznih grupa identiteta Mediterana s točke gledišta ovoga austrijskog diplomata i pisca.
\end{abstract}

Ključne riječi: Europa; Sredozemlje; Orijent; identitet; religij 